\title{
Biochemical and Physiological Basis for Treating Hydrogen Gas as a Medicine
}

\author{
Yuri Pivovarenko \\ Research and Training Centre Physical and Chemical Materials Science, Kyiv Taras Shevchenko University and NAS of Ukraine, Kiev, \\ Ukraine \\ Email address: \\ y.pivovarenko@gmail.com \\ To cite this article: \\ Yuri Pivovarenko. Biochemical and Physiological Basis for Treating Hydrogen Gas as a Medicine. European Journal of Preventive \\ Medicine. Vol. 7, No. 6, 2019, pp. 100-107. doi: 10.11648/j.ejpm.20190706.12
}

Received: October 7, 2019; Accepted: October 29, 2019; Published: November 6, 2019

\begin{abstract}
Due to the activity of normal microflora, gaseous hydrogen is constantly formed in the human intestine. Thus, the constant contact of the human body with gaseous hydrogen is absolutely natural. One of the consequences of this contact is that the internal organs of a person are in a peculiar gas gradient formed, on the one hand, by gaseous hydrogen of intestinal origin, and on the other, by gaseous oxygen of air. Since gaseous hydrogen is an electron donor in an aqueous medium, and gaseous oxygen is their typical acceptor, the internal organs of a person find themselves in an electrostatic field, the intensity of which depends mainly on the content of hydrogen gas in the intestine. Despite the fact that this is practically obvious, the influence of such an electrostatic field on the state of the internal organs of a person, in particular, on the movement of ions in them, is usually not taken into account. Also, the exceptional penetrating power of hydrogen gas is not taken into account, due to which it can penetrate from the human intestine into the cells of other tissues and replace typical biological oxidation substrates in them. Thus, it can be expected that by controlling the formation of gaseous hydrogen in the intestine, one can also control the activity of human metabolism. For this reason, various methods that can increase the content of gaseous hydrogen in the intestines of a person may be of medical interest. Also, doctors may be interested in those sources of atomic hydrogen, the use of which can increase its content in other parts of the human body, in particular, some alternative medicine. All of this is discussed here. In addition, the medical significance of carbon dioxide, which initiates the decomposition of water into atomic hydrogen and molecular oxygen, is also analyzed here.
\end{abstract}

Keywords: Hydrogen, Carbon Dioxide, Carbon Dioxide, Acupuncture, Amber

\section{Introduction}

Due to the activity of normal microflora, the proportion of gaseous hydrogen in the intestinal gas of a person is usually $\sim 20 \%$ (vol.), but may be 2 to 3 times this value. At the same time, the usual content of gaseous oxygen in the human intestine is about $0 \%$ (vol.) [1]. Given the opposite effect of hydrogen gas and oxygen on the hydration of biopolymers (Figure 1) [2], it can be assumed that a high content of hydrogen gas in the intestine (in the complete absence of gaseous oxygen) contributes to the dehydration of biopolymers, which are the main components of nutrients.

Thus, we can conclude that the high content of gaseous hydrogen in the human intestine provides facilitated contact of the digestive enzymes with food polymers cleaved by them and thus contributes to the activation of digestion.
In addition, due to the high content of gaseous hydrogen in the human intestine (which is comparable to the oxygen content in the air [3]), the human body can be represented as a kind of air-hydrogen electrochemical cell (Figure 2) [4].

For this reason, the internal organs of a person are constantly affected by an electrostatic field, the intensity of which is determined mainly by the content of gaseous hydrogen in the intestine and which distributes the ions present in the tissues of the human body.

In this regard, one should be aware that such an electric field can be of paramount importance for human digestion, given that most of the nutrients that enter the intestine or are formed in it have or acquire an anionic nature: fatty and organic acids, amino acids (with $\mathrm{pH} \sim 8$ ), glucose phosphate [4]. Thus, taking into account the direction of the described electric field (Figure 2), it can be assumed that the anionic 
nature of nutrients is very important for their transfer to the blood and, as a result, to other organs, including the skin.

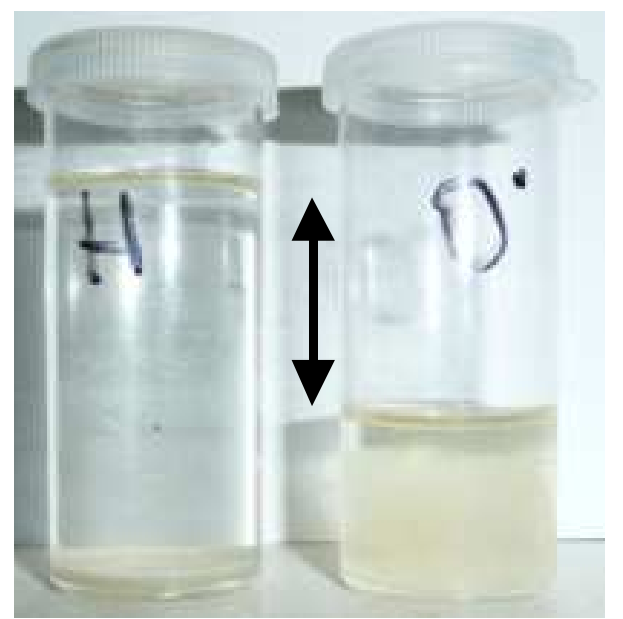

Figure 1. This is the swelling of starch, which is the main food biopolymer in the human diet. You can see that starch does not swell in water that is saturated with hydrogen (left), but swells in water that is saturated with oxygen (right); it should be noted that in water that is saturated with hydrogen (left), starch does not swell for months [2].

Water saturated with oxygen can evaporate even from a closed plastic bottle: the arrow shows how much the level of such water has decreased during the day; water gets the same permeability under the influence of ionizing radiation [5].

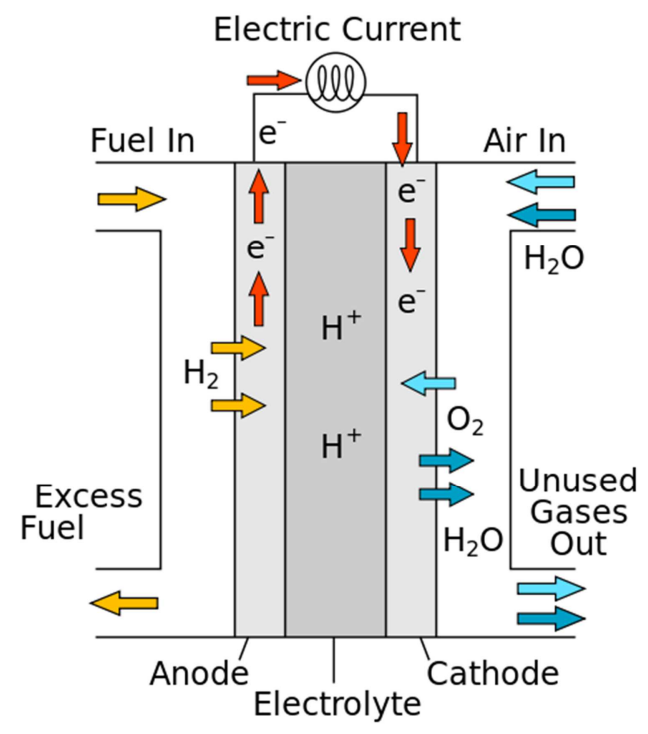

Figure 2. This is the scheme of an air-hydrogen electrochemical cell [3, 4]. Red arrows indicate the movement of electrons from the compartment with an aqueous solution saturated with hydrogen gas, into the compartment with an aqueous solution saturated with air. Anionic nutrients can, like electrons, travel through the intestinal mucosa in contact with hydrogen gas to the surface of the skin and lungs that are in contact with air [3].

It is also clear why this directional electric field is capable of blocking the exit of cations such as $\mathrm{Ca}^{2+}, \mathrm{Mg}^{2+}, \mathrm{Fe}^{2+}$ and $\mathrm{Fe}^{3+}$ from the intestine into the blood. Moreover, this same field allows one to explain why all these ions are able to penetrate from the intestines into the blood in the form of salts of citric, phosphate or orthophosphate acid, the anions of which carry a significant negative charge. In addition, given such an electric field, one can understand why the deficit of such cations in the human body can be filled with the help of therapeutic muds and bathing in mineral springs, especially in combination with an increase in the content of hydrogen gas in the intestines.

It should be noted that the value of intestinal gaseous hydrogen is not limited to the formation of the described electric field. So, given the abnormally high penetrating power of hydrogen gas (Figure 3), it can be expected that it can penetrate from the intestine into all tissues of the human body.

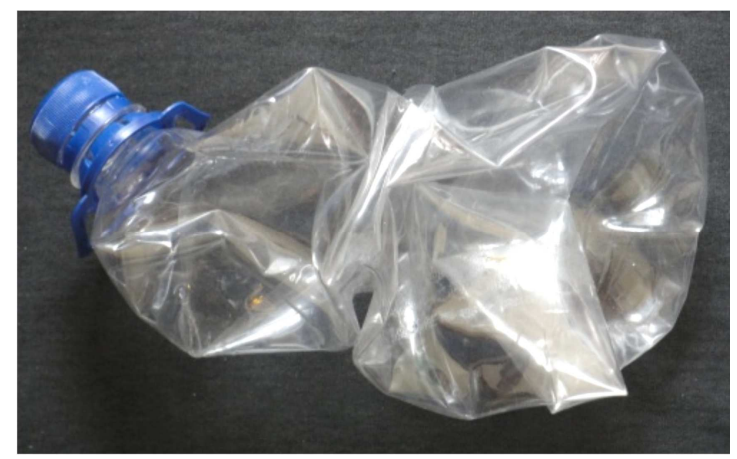

Figure 3. Demonstration of high permeability of hydrogen gas: PET bottle with gaseous hydrogen 4 weeks after filling [3]. Gaseous oxygen does not have such penetration.

Given the penetration of gaseous hydrogen, it can be assumed that gaseous hydrogen of intestinal origin can penetrate even into the cytoplasm and mitochondria. This, in turn, suggests its direct participation in the chemical reduction of such transport forms of atomic hydrogen as NAD (Nicotinamide Adenine Dinucleotide), NADP (Nicotinamide Adenine Dinucleotide Phosphate) and FAD (Flavin Adenine Dinucleotide), in other words, it suggests the direct participation of gaseous hydrogen gas of intestinal origin in glycolysis, $\alpha-, \beta$-, and $\omega$-oxidation of fatty acids, Krebs cycle, etc.

Here, some considerations need to be made. Commonly used schematic representations of the Krebs cycle contain many details (Figure 4). For this reason, it is not always clear that the main products of the Krebs cycle are hydrogen atoms (Figure 5).

Such clarity is important because it makes it possible to conclude that atomic hydrogen is the ultimate goal of the breakdown of nutrients in the human diet. (All this also applies to all processes in which biological oxidation occurs, coupled with the formation of NADH, NADPH and $\mathrm{FADH}_{2}$ [4]).

For the same reason, it is not always clear that the Krebs cycle is an active generator of carbon dioxide (Figure 5), which is usually taken as a by-product. This point of view may be fundamentally wrong, given that carbon dioxide is the initiator of the decomposition of water into atomic hydrogen and molecular oxygen [6]:

$4 \mathrm{H}_{2} \mathrm{O}+4 \mathrm{CO}_{2} \rightarrow 4 \mathrm{H}_{2} \mathrm{CO}_{3} \rightarrow 2 \mathrm{H}_{2} \mathrm{O}+4 \mathrm{CO}_{2}+\mathrm{O}_{2}+4 \mathrm{H}^{*}$

Given all this, let's assume that carbon dioxide is also necessary for humans. 


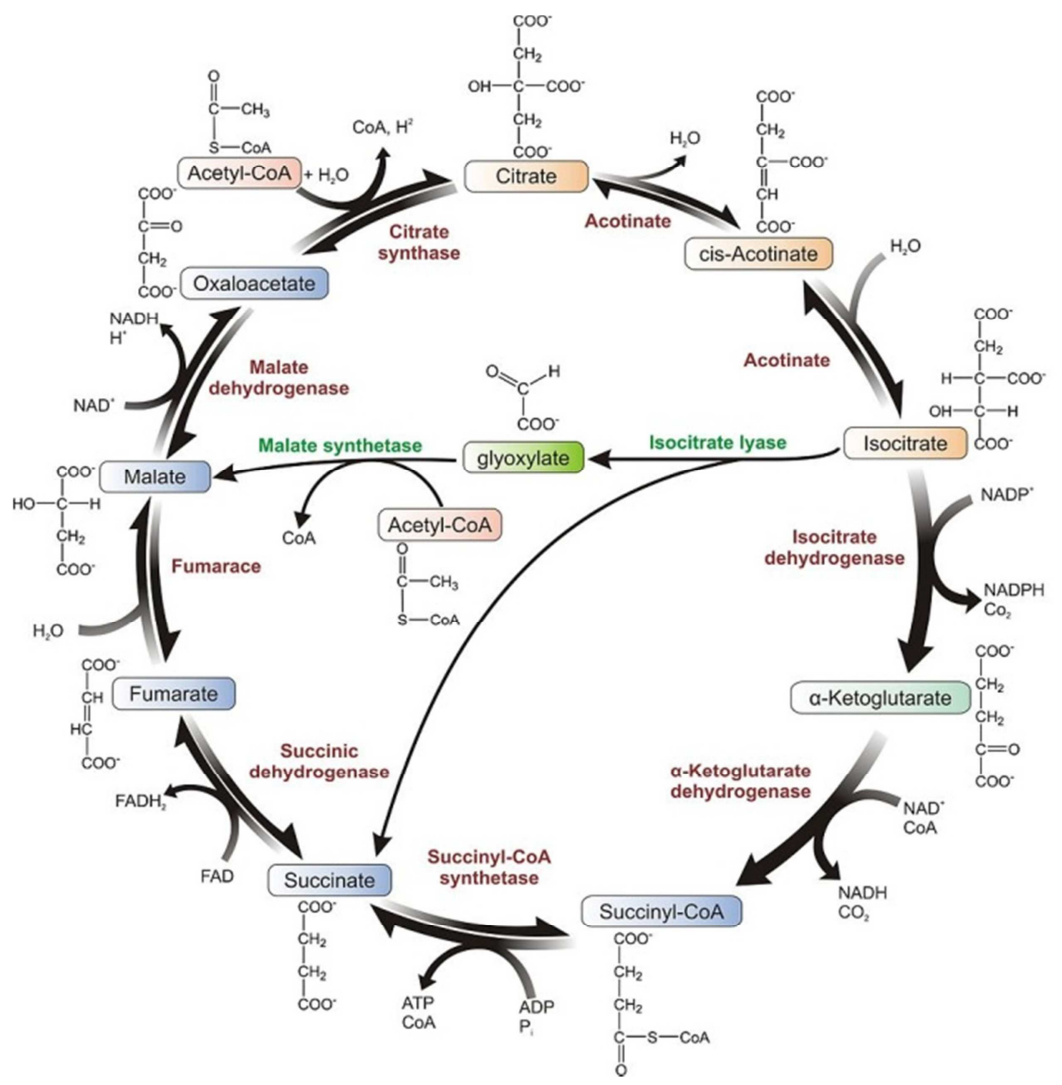

Figure 4. This is a typical Krebs cycle diagram.

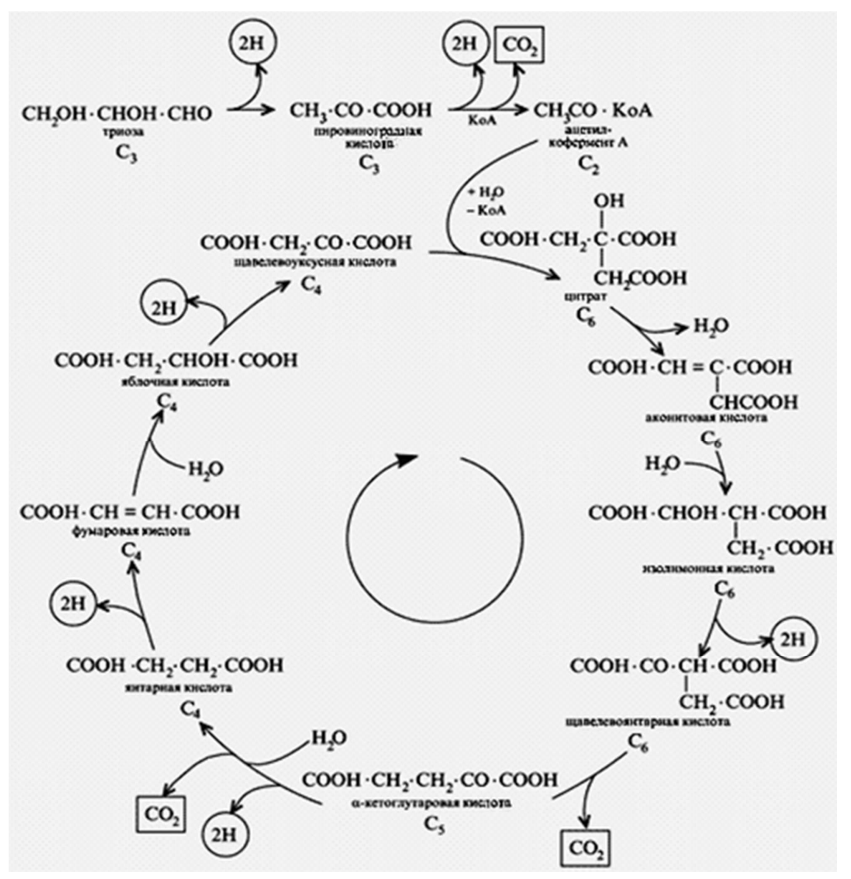

Figure 5. From the schemes of the Krebs cycle that I studied, the Russianlanguage scheme turned out to be the most suitable for this article. Pairs of hydrogen atoms released in the reactions of the cycle and participating in the reduction of NAD and FAD are circled, and the molecules of carbon dioxide released are enclosed in squares [4].

Taking all this into account, let's analyze in detail the medical prospects for using both of these gases, as well as their sources, which are most suitable for medical practice.

\section{Main Body}

Agree that after such an argument it is advisable to analyze at least some ways to increase the atomic hydrogen content in the human body.

\subsection{Sources That can Increase Gaseous Hydrogen in the Intestines}

\subsubsection{Suitable Intestinal Microflora}

Given that gaseous hydrogen appears in the human intestine due to the activity of normal intestinal microflora, its oral use can be recommended as the most natural way to increase the content of hydrogen gas in the human intestine.

Despite its naturalness, the application of this method can cause difficulties, since the ability of intestinal microflora to generate gaseous hydrogen depends on the human diet, in fact - the diet of intestinal microflora [7, 8]. Thus, this method can be associated with additional and expensive medical research. (In addition, one should take into account the possible effect of intestinal microflora on the epigenetic activity and structure of the human genome [9], as well as on the absence in an adult of an immunological tolerance to non-characteristic types of intestinal microflora [10]) For this reason, more acceptable ways to increase the gaseous hydrogen content in the intestine will be discussed below. 


\subsubsection{Catholyte}

Probably the most popular of these methods is the use of catholyte, i.e. - a solution formed in the cathode compartment of the electrolyser. I should be noted that the freshly prepared catholyte is enriched precisely with atomic hydrogen (in situ). Therefore, it is not surprising that it is freshly prepared catholyte that is an effective stimulator of biochemical and physiological processes in the human body. Moreover, through the oral use of catholyte, a person becomes less sensitive to heat, cold and radiation. This is due to the fact that catholyte is not bound by the biopolymers that make up the human body, just like starch itself (Figure 1, left). Therefore, with the oral use of catholyte, the human body gets rid of excess water (in particular, edema), which increases its sensitivity to the action of these adverse factors.

Explaining the stimulating effect of catholyte on the human body, it is also necessary to take into account its ability to actively absorb, thanks to the alkaline reaction, carbon dioxide, which is the initiator of the decomposition of water into atomic hydrogen and molecular oxygen (1). In this regard, it should be noted that the saturation of catholyte with carbon dioxide is usually not taken into account, including by specialists who seek to explain its therapeutic effect, which is fundamentally wrong.

\subsection{3. "Magnesium" Water}

It is well known that hydrogen atoms are released during the reaction of water with metallic magnesium [3]:

$$
\mathrm{Mg}+2 \mathrm{H}_{2} \mathrm{O} \rightarrow \mathrm{Mg}(\mathrm{OH})_{2}+2 \mathrm{H}^{*}
$$

Thus, "magnesium water", like freshly prepared catholyte, is a solution saturated with atomic hydrogen in situ. For this reason, "magnesium water" should be considered not only as a source of magnesium ions, but also of atomic hydrogen, and its therapeutic properties should be explained accordingly (not forgetting, of course, about its alkaline reaction).

\subsubsection{Other Available Sources that can Increase the Amount of Gaseous Hydrogen in the Human Intestines}

It should be noted that there are other, sometimes quite peculiar, sources with which you can increase the content of gaseous hydrogen in the human intestines. So, relatively recently, Soviet people received aqueous solutions of atomic hydrogen, "charging" water with the help of TVs of old models. In fact, such charging was carried out thanks to the electrons emitted by the picture tubes - such electrons fly off the screen by $\sim 2$ meters. Interacting with aqueous hydrogen ions, such electrons transformed them into atomic hydrogen:

$$
\mathrm{e}^{-}+\mathrm{H}^{+} \rightarrow \mathrm{H}^{*}
$$

Since water vapor is always positively charged [11], the same negatively charged water can be obtained by evaporating it in the dark - light causes a positive electrization of water [12]. One can hear about the healing properties of such "settled" or "separated" water, as well as about the healing properties of the water "sanctified" in the church, which is customary to keep for a long time.

In addition, water that is in contact with ice (in particular, "melt water") is also enriched with atomic hydrogen: since the dielectric constants of water and ice in thermal equilibrium (at $0^{\circ} \mathrm{C}$ ) are $\sim 88.3$ and $\sim 99,3$, respectively [3], water, according to the rule of Kyon [3], is always negatively charged, and ice is positive. (Usually, with such a contact, the electric potential of ice relative to water does not exceed $+10 \mathrm{~V}$, however, values greater than $+100 \mathrm{~V}$ were also recorded [13])

Obviously, the list of methods for producing water enriched in atomic hydrogen can be continued, but we will limit ourselves to those mentioned. Given this diversity, methods for determining the activity of atomic hydrogen in aqueous solutions can be very important for practitioners.

\subsubsection{Atomic Hydrogen Fluorescence in Aqueous Media as a Promising Way to Determine Its Activity}

It was previously established that the fluorescence spectra of aqueous solutions containing various sources of atomic hydrogen have peaks with maxima at wavelengths that well coincide with the lines of the Ballmer series [14, 15]. For example, such a peak is clearly visible on the fluorescence spectrum of an aqueous solution of formic acid containing zinc oxide (Figure 6) - it is known that zinc oxide catalyzes the decomposition of formic acid into two hydrogen atoms and a carbon dioxide molecule [3]:

$$
\mathrm{HCOOH} \rightarrow \mathrm{CO}_{2}+2 \mathrm{H}^{*}
$$

As can be seen (Figure 6), this decomposition manifests itself in the appearance of a fluorescence peak with a maximum near the short-wavelength boundary of the Ballmer series (364.56 nm) [16].

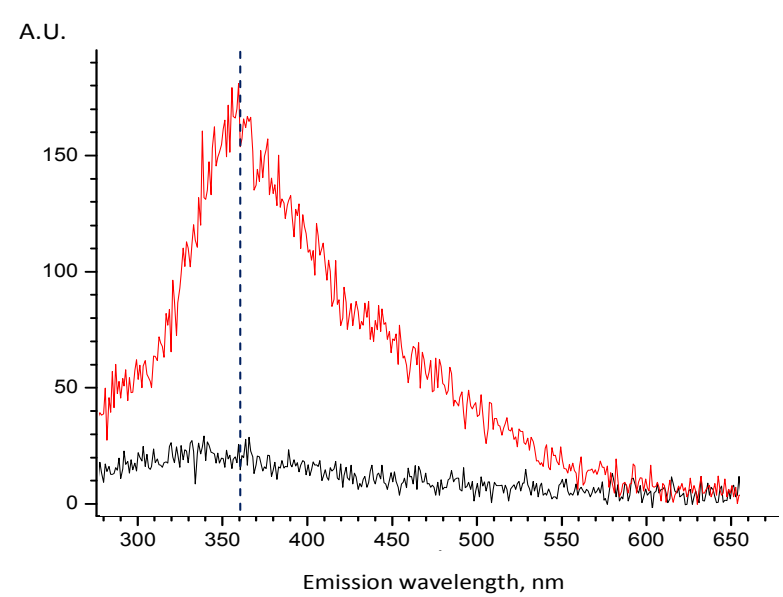

Figure 6. These are the fluorescence spectra of distilled water (black curve) and aqueous solutions containing products of decomposition of formic acid: $\mathrm{HCOOH} \rightarrow \mathrm{CO}_{2}+2 \mathrm{H}^{*}$ (red curve). Such decomposition is immediately accompanied by the appearance of a peak of fluorescence with a maximum (blue dotted line) near the Ballmer series shortwave border (364.56 nm) [16].

Since the existence of this kind of fluorescence has been repeatedly confirmed experimentally, it can be recommended as a convenient way to determine the activity of atomic hydrogen in aqueous media, including biological ones. 
The excitation wavelength of the fluorescence was 260 $\mathrm{nm}$. The working solution was at room temperature throughout the experiment.

Since this is important for the completeness of the description, it should be noted that this type of fluorescence is excited by UV radiation in the range of $180-320 \mathrm{~nm}$, and with almost the same efficiency. Thus, this radiation is a typical fluorescence, since it can be initiated by light of different wavelengths.

\subsection{Inhaled Hydrogen Gas}

Recently, videos showing the thrombolytic effect of inhaled gaseous hydrogen, which releases in the cathode compartment of the electrolyzer, have been demonstrated on the Internet (keywords to search: instantaneous therapeutic effect of hydrogen). So, it is shown that such an inhalation is accompanied by the rapid, within one to two minutes, dissolution of blood clots in the subcutaneous capillaries of the fingers. Since it is not only spectaculars, but also important, this action of inhaled hydrogen gas must be analyzed.

It is obvious that such hydrogen, which is inhaled immediately after its release in the cathode compartment of the electrolyzer (in fact, in situ), contains a significant amount of atomic hydrogen. Thus, taking into account the results obtained in studying the swelling of biopolymers in aqueous solutions electrized by passing gaseous hydrogen and oxygen (Figure 1) [2], it can be expected that the flow of gaseous hydrogen into the blood will contribute to the dehydration of its components and therefore degrade their adhesive properties.

The result of such an impact will be the defragmentation of blood clots and their peeling from the walls of the capillaries, which, in fact, show advertising videos.

Analyzing the proposed content, one should take into account the slow circulation of blood in the capillaries of the fingers, one of the most distant parts of the circulatory system from the heart. You should also consider the contact of the subcutaneous capillaries with oxygen. These features cause the susceptibility of subcutaneous capillaries of fingers to thrombus formation. At the same time, the analysis of the proposed content suggests that if inhalation of gaseous hydrogen leads to the destruction of blood clots even in the subcutaneous capillaries of the fingers, we can expect even more pronounced thrombolytic effects of inhaled gaseous hydrogen on the blood vessels less distant from the heart.

This confirms the expectations that gaseous hydrogen also has no barriers when moving through the human body, as well as when moving through plastic (Figure 1). Be that as it may, the video being analyzed requires that the fastest flow of hydrogen gas into the blood occurs through the lungs, which is not surprising given their saturation with capillaries.

By analyzing the described effect of inhaled gaseous hydrogen on the state of subcutaneous capillaries, one can expect activation of the metabolism in the skin and, as a result, its rejuvenation. This is of fundamental importance, since we are used to assessing the age of a person precisely by the condition of his skin.

\subsection{Other Sources of Gaseous Hydrogen in the Skin}

\subsubsection{Metallotherapy}

Another method of increasing the concentration of atomic hydrogen in human skin is metal therapy, which usually means the use of applications of metallic copper and copper alloys for medicinal purposes. So, already in ancient literary sources it was reported that diseases of the skin, eyes, hearing organs, inflammation of the tonsils, radiculitis, polyarthritis, bruises and bone fractures, epilepsy and even cholera prophylaxis can be treated using applications of metallic copper and its alloys. According to later sources, applications of metallic copper and copper alloys relieve pain, normalize blood pressure, and help with respiratory diseases (sinusitis, frontitis, rhinitis, otitis media and bronchitis), tuberculosis, radiculitis, hemorrhoids, postoperative hernias, kidney stones, diabetes and improve metabolism. Relatively recently, it was shown that such applications lower the temperature, have a hemostatic effect, promote resorption of subcutaneous hemorrhages, edema, tumors, reduce tinnitus, restore hearing, suppress inflammation of the urinary and gall bladders, kidneys, lungs, and organs of the cardiovascular system, improve post-infarction and postoperative condition and, as expected, help in the treatment of skin diseases.

Analyzing the described therapeutic effects, I suggested that they are due to the electron-donating properties of metallic copper and its alloys with respect to aqueous media and, therefore, with their ability to enrich aqueous media with hydrogen atoms, as in (2). Subsequently, this assumption was confirmed by me in a number of experiments. I want to bring to your attention the most significant, in the aspect of this work, experimental confirmation of this assumption. Thus, a convincing manifestation of the electron-donating properties of metallic copper and its alloys was observed when studying the UV absorption spectra of aqueous DNA solutions that were in contact with metallic copper or its alloys. So, it was found that peaks with a maximum at a wavelength of $260 \mathrm{~nm}$ can completely disappear from such spectra. To well understand the significance of such disappearance, two facts must be taken into account. First, we must take into account that oxygenation of aqueous DNA solutions is accompanied by an increase in this peak, and their deoxygenation is accompanied by its decrease [17, 18]. Secondly, it is necessary to take into account the complete disappearance of these peaks after saturation of aqueous DNA solutions with hydrogen gas $[19,20]$. All this proves that, in relation to aqueous DNA, metallic copper and copper alloys are the same oxygen antagonists as hydrogen gas. Thus, they can exhibit electron-donating properties with respect to aqueous media and, therefore, initiate the formation of atomic hydrogen in them, as shown in in (2).

The described results should not be taken simply as an interesting phenomenon. So, analyzing these results, it is advisable to take into account a significant peak with a maximum at a wavelength of $260 \mathrm{~nm}$ in the UV absorption 
spectra of suspensions of B-lymphocytes of patients with chronic B-cell chronic lymphocytic leukemia and the absence of such a peak in similar spectra of healthy people (Figure 7) [20].

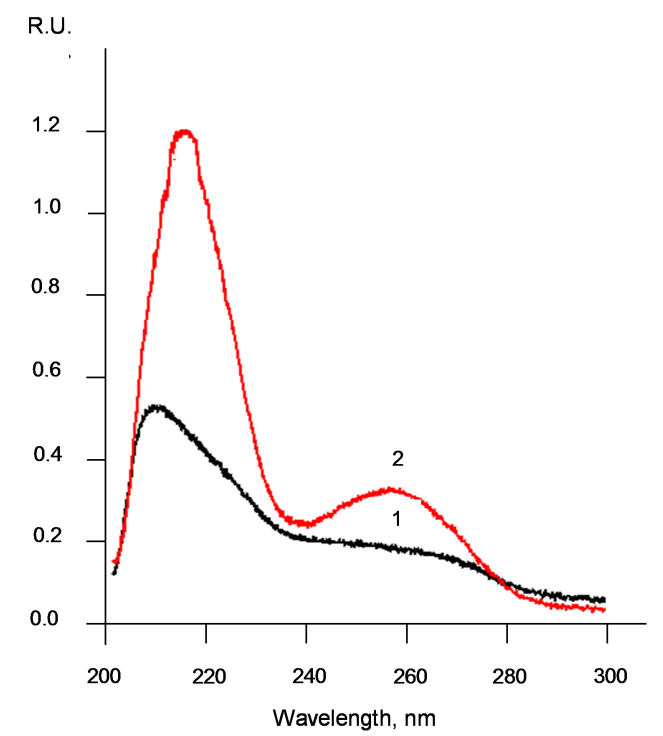

Figure 7. UV absorption spectra of B-lymphocyte suspensions: 1 - healthy person; 2 - patient with chronic B-cell chronic lymphocytic leukaemia [20].

Analyzing the proposed mechanism of the therapeutic effect of metallic copper and copper alloys, it is also necessary to take into account that it is oxidative damage to DNA that is recognized as the main cause of arthritis, multiple sclerosis, a number of neurological disorders, some forms of cancer, as well as diseases of the heart, gastrointestinal tract, Alzheimer's and Parkinson's [21 - 25]. Thus, the deoxygenating effect of metallic copper and its alloys on DNA allows them to be included in the number of agents that can repair oxidative damage to DNA and, therefore, contribute to the cure of many diseases, including intractable ones.

Since active oxygen is a universal activator of cell proliferation [26, 27], the bacteriostatic and antiviral activity of metallic copper and its alloys can also be explained by the fact that atomic hydrogen is a chemical antagonist of active oxygen [3].

Also during such experiments, it was found that metallic aluminum has more pronounced electron-donating properties than metallic copper with respect to aqueous media, naturally. This in itself is not surprising, given the position of copper and aluminum in the electrochemical series of voltages [3]. However, this allows us to explain why the use of copper-aluminum alloys gives the most pronounced therapeutic effect. In addition, this allows us to recommend food aluminum as a more promising metal therapy compared to metallic copper.

\subsubsection{Acupuncture}

Formally, acupuncture can be considered as a type of metallotherapy. At the same time, with acupuncture, an additional, in comparison with metal therapy, mechanism of saturation of reflex points with electrons and, as a result, atomic hydrogen is realized. To understand the essence of such a mechanism, one should take into account the polarization of terrestrial objects, the idea of which is provided by the polarization of clouds (Figure 8) [28] and the standard position of the needles on the patient's body (Figure 9).

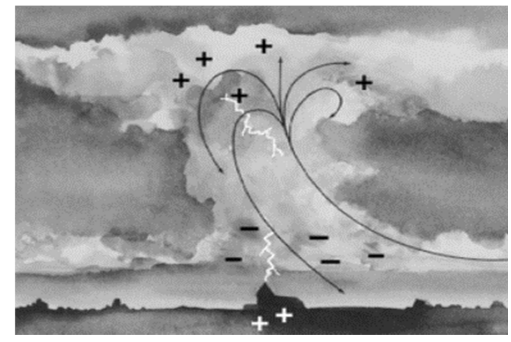

Figure 8. Polarization of clouds: the lower part of a typical cloud has a negative charge and the upper part has a positive charge; all terrestrial objects are polarized in the same manner [28].

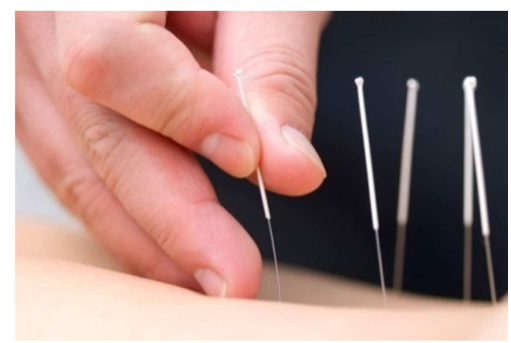

Figure 9. The lower tips of the needles are places with an increased density of electrons that flow into the patient's body as from a lightning rod.

Such polarization allows us to conclude that the tip of the needle has an increased density of electrons "flowing" into the patient's body in the same way as electrons flow into the earth from a lightning rod. It is clear that the intensity of such a "sink" of electrons can be increased by increasing the temperature of the needles, that is, due to the thermal emission of electrons. Thus, the therapeutic effect of acupuncture can also be explained by the enrichment of reflex points with atomic hydrogen, which is formed as in (2). (Agree, such a look at acupuncture is quite unexpected.)

Thus, it can be assumed that:

1. The therapeutic effects of metallotherapy as a whole, including acupuncture, are due to the electron-donating and, as a result, hydrogen donating, properties of the metals used, in fact - their antioxidant properties.

2. To realize the therapeutic effect of metallotherapy on human internal organs, the ability of hydrogen atoms to penetrate deep into the human body from the surface of the skin is of fundamental importance.

Of course, in explaining the therapeutic effects of metallotherapy, one should also consider the opposite effect of gaseous hydrogen and oxygen on the ability of water to hydrate biopolymers (Figure 1). Since cell division is inevitably accompanied by the accumulation of water, which is the main substance of living objects, it can be assumed that gaseous oxygen stimulates this accumulation, and gaseous hydrogen blocks it. 


\subsection{Amber}

It is likely that the most elegant methods to increase the concentration of atomic hydrogen in the skin, in particular in the subcutaneous capillaries, are associated with the use of amber jewelry. So, given the pronounced ability of amber to adsorb electrons, it can be expected that the skin that comes into contact with the amber jewelry receives a portion of the electrons pre-sorbed by the amber surface. Thus, wearing jewelry made of this stone may be accompanied by an increase in the atomic hydrogen content in the blood of skin capillaries, in accordance with (2).

\subsection{Pulsed Electromagnetic Fields}

As you know, pulsed low-frequency electromagnetic fields (PEMFs) can stimulate the restoration of damaged tissues, including their innervation. Despite the fact that the restorative effect of such fields has long been used in medicine, its nature remained unknown [29]. Recently, I proposed the following explanation for the therapeutic effect of PEMFs: by inducing electrolysis in the tissues of the human body, PEMFs enrich it with electrolysis products, in particular atomic hydrogen [30]. Given the deep penetration of PEMFs into the tissues of the human body, we can assume that they are able to saturate with atomic hydrogen all the compartments of the cells of the human body, including cell nuclei. This allows us to explain both the physiological and molecular genetic effects of the described PEMFs.

\subsection{Baking Soda and Other Sources of $\mathrm{CO}_{2}$}

There is an opinion that aqueous solutions of baking soda (sodium bicarbonate) can be used as a prophylactic agent that can prevent the appearance of a number of diseases, including cancer, and in some cases - get rid of them. However, in the opinion of opponents, baking soda water solutions should be used exclusively to reduce the acidity of gastric juice or disinfection. As a rule, the arguments presented by the participants of this, more often virtual, discussion, do not convince their opponents.

I will try to support the "supporters" of baking soda, using as an argument the ability of carbon dioxide to initiate the decomposition of water into atomic hydrogen and molecular oxygen, in accordance with (1) [6].

Thus, baking soda, taken orally or used externally, can be considered as a source of atomic hydrogen, which explains its healing properties. (It becomes clear why it is not easy for people to abandon bakery products and beer made using yeast and bicarbonates.)

Plants whose enzymatic systems are oriented towards its active assimilation should be mentioned as oral sources of $\mathrm{CO}_{2}$ (this is an argument in favor of supporters of vegetarianism).

Obviously, the same reasoning is applicable to explain the therapeutic effects of methods for increasing $\mathrm{CO}_{2}$ concentrations in the blood, such as breathing exercises associated with breath holding. Considering (2), it can be assumed that the mechanism of the therapeutic effect of such exercises is identical to the mechanism of the therapeutic effect of inhaled gaseous hydrogen. (At the same time, it becomes clear why it is not easy for people to give up smoking, which is a kind of source of $\mathrm{CO}_{2}$ for them.)

All this must be taken into account in the medical use of carbon dioxide anhydrase inhibitors [31], enzymes that accelerate the formation of carbon dioxide:

$$
\mathrm{H}_{2} \mathrm{O}+\mathrm{CO}_{2} \rightarrow \mathrm{H}_{2} \mathrm{CO}_{3}
$$

In other words, it must be borne in mind that the inhibition of carbonic anhydrase is accompanied by the simultaneous depletion of human tissues with atomic hydrogen.

\section{Conclusion}

Given the importance of atomic hydrogen for human bioenergetics, it can be considered as a natural stimulator of human metabolism. Thus, medical methods, the purpose of which is, in fact, an increase in the content of atomic hydrogen in the human body, are quite reasonable.

Since $\mathrm{CO}_{2}$ initiates the decomposition of water, accompanied by the release of atomic hydrogen, medical methods aimed at increasing the $\mathrm{CO}_{2}$ content in the human body also seem to be quite reasonable. Since atomic hydrogen and $\mathrm{CO}_{2}$ are normal metabolites of the human body, their medical use seems more attractive than the use of medications that are unnatural for humans, including synthetic ones.

Given the generally accepted culture of biological research, it can be argued that the study of most biological objects was carried out in environments lacking atomic hydrogen, moreover, in environments with an uncontrolled content of air and gaseous oxygen. Therefore, the properties of most biological objects in vitro can differ significantly from their properties in vivo and, therefore, cannot be extrapolated directly to living organisms. Since this situation cannot be considered satisfactory, the properties of biological objects should also be studied in environments containing atomic hydrogen and not containing oxygen and air.

\section{References}

[1] N. V. Harchenko, V. V. Tchernenko, I. N. Tchervak, V. M. Stukalo and D. V. Tokar (2008) Using Espumizan preparation in practice gastroenterologist. Ukraine's Health, 6, 52-53. In Russian.

[2] Y. Pivovarenko (2018) \pm Water: Demonstration of water properties, depending on its electrical potential. World Journal of Applied Physics, 3 (1), 13-18.

[3] B. V. Nekrasov (1974) Basics of General Chemistry, 1. Moscow: Chemistry, 656. In Russian.

[4] Y. Pivovarenko (2018) The value of gaseous hydrogen generated by the intestinal microflora of human. Chapter 7 in: Top 10 Contributions on Biomedical Sciences, 2-15. Hyderabad, India: Avid Science. 
[5] J. E. Spangenberg and T. W. Vennemann (2008) The stable hydrogen and oxygen isotope variation of water stored in polyethylene terephthalate (PET) bottles. Rapid Commun. Mass Spectrom., 22, 672-676.

[6] Yu. V. Pletner and V. S. Poloskin (1977) Workshop on the methodology of teaching chemistry. Moscow: Enlightenment, 206. In Russian.

[7] M. C. Collado, E. Isolauri and K. Laitinen (2008) Distinct composition of gut microbiota during pregnancy in overweight and normal-weight women. Am. J. Clin. Nutr., 88, 894-899.

[8] J. K. Di Baise, H. Zhang, M. D. Crowell et al. (2008) Gut microbiota and its relationship with obesity. Mayo Clin. Proc., 83 (4), 460-469.

[9] Ye. A. Korniyenko (2013) Current Conception of the link of obesity and intestinal microbiota. Pediatr, 4 (3), 3-14.

[10] R. Sharma, C. Young and J. Neu (2010) Molecular modulation of intestinal epithelial barrier: contribution of microbiota. J. Biomed. Biotech, 10. 1155/305879, on line.

[11] N. V. Krasnogorskaya (1984) Electromagnetic fields in the atmosphere of the Earth and their biological significance, 1. Moscow: Nauka, 377. In Russian.

[12] F. Crawford (1968) Waves; BPC, 3. NY: McGraw-Hill, 600.

[13] V. M. Muchnik (1974) Physics of thunderstorms. Leningrad: Hydrometeoizdat, 351. In Russian.

[14] Y. Pivovarenko (2017) An alternative strategy in cancer chemotherapy, aimed not at killing cancer cells, but the recovery of their DNA, modified by active oxygen. Biomedical Sciences, 3 (5), 94-98.

[15] Y. Pivovarenko (2019) Laser-Induced Fluorescence of Wet Porous Silicon as Laser-Induced Fluorescence of $\mathrm{H}_{3} \mathrm{O}^{+}$. Journal of Photonic Materials and Technology, 5 (1), 11-15.

[16] E. V. Shpolsky (1974) Atomic Physics, 1. Moscow: Nauka, 576. In Russian.

[17] R. Doshi, P. J. R. Day and N. Tirelli (2009) Dissolved Oxygen Alteration of the Spectrophotometric Analysis and Quantication of Nucleic Acid Solutions. Biochemical Society Transactions, 37 (2), 466-470.

[18] R. Doshi, P. J. R. Day, P. Carampin at all. (2010) Spectrophotometric analysis of nucleic acids: Oxygenationdependant hyperchromism of DNA. Anal. Bioanal. Chem., 396, 2331-2339.
[19] Yu. V. Pivovarenko (2014) Hypochromism of Degas DNA Solutions. Modern High Technologies, 3, 147-149.

[20] Y. Terentyeva and Y. Pivovarenko (2015) UV Absorbance of Lymphocytes. European Journal of Advanced Research in Biological and Life Sciences, 3 (4), 20-24.

[21] T. Ozawa (1997) Oxidative Damage and Fragmentation of Mitochondrial DNA in Cellular Apoptosis. Bioscience Reports, 17 (3), 237-250.

[22] H. E. Poulsen, H. Prieme and S. Loft (1998) Role of oxidative DNA damage in cancer initiation and promotion. Eur. J. Cancer Prev., 7, 9-16.

[23] E. Cadenas and K. J. A. Davies (2000) Mitochondrial free radical generation, oxidative stress and aging. Free Radic. Biol. Med., 29, 222-230.

[24] M. Cooke, M. D. Evans, M. Dizdaroglu and J. Lunec (2003) Oxidative DNA damage: mechanisms, mutation, and disease. FASEB, 17, 1195-1214.

[25] J. Cadet and K. J. A. Davies (2017) Oxidative DNA Damage \& Repair: An Introduction. Free Radicals Biology and Medicine, 107, 2-12.

[26] R. H. Burdon (1995) Superoxide and hydrogen peroxide in relation to mammalian cell proliferation. Free Radic. Biol. Med, 28, 463-499.

[27] R. G. Allen and M. Tresini (2000) Oxidative stress and gene regulation. Free Radic. Biol. Med., 28, 463-499.

[28] Y. Pivovarenko (2018) The Nature of the Celestial Elves, Sprites and Jets. Discovery Nature, 12, 1-4.

[29] T. J. Goodwin (2003) Physiological and Molecular Genetic Effects of Time-Varying Electromagnetic Fields on Human Neuronal Cells; Technical Report of NASA Johnson Space Center; Houston, TX, United States, 38.

[30] Y. Pivovarenko (2019) Arborization of Aqueous Chlorides in Pulsed Electromagnetic Fields as a Justification of their Ability to Initiate the Formation of New Neuronal Dendrites. International Journal of Neurologic Physical Therapy, 5 (1), 21-24.

[31] P. Koutnik, E. G. Shcherbakova, S. Gozem, M. G. Caglayan, T. Minami and P. Anzenbacher, Jr. (2017) Fluorescence-Based Assay for Carbonic Anhydrase Inhibitors. Chem, 2, 271-282. 